\title{
Infográficos versus Materiais de Aprendizagem Tradicionais: uma Investigação Empírica
}

\author{
Kamila T. Lyra - Universidade de São Paulo (ICMC-USP) - kamilalyra@ gmail.com \\ Brauner R. N. Oliveira - Universidade de São Paulo (ICMC-USP) - brauner@ usp.br \\ Rachel C. D. Reis - UFV, Universidade de São Paulo (ICMC-USP) - rachel.reis@ufv.br \\ Wilmax M. Cruz - Universidade de São Paulo (ICMC-USP) - wilmaxmcruz@ gmail.com \\ Elisa Y. Nakagawa - Universidade de São Paulo (ICMC-USP) - elisa@icmc.usp.br \\ Seiji Isotani - Universidade de São Paulo (ICMC-USP) - sisotani @icmc.usp.br
}

Resumo - Infográfico é um tipo popular de visualização de informação que utiliza de apelo visual para tornar a transmissão de informação rápida e efetiva. No entanto, ainda existem poucos trabalhos empíricos que investiguem sua interferência no processo de aprendizagem e interação com as características estilos de aprendizagem e satisfação. Nesse contexto, este trabalho teve como objetivo avaliar a eficácia dos infográficos em comparação com os materiais de aprendizagem tradicionais (gráfico e texto). Para isso, foi executado um experimento com 54 alunos de graduação. Os alunos foram distribuídos entre os três tipos de materiais e avaliados em relação ao aprendizado, retenção de conhecimento e satisfação pessoal. Os resultados mostraram que os infográficos foram tão bons para a aprendizagem e retenção de conhecimento quanto os materiais tradicionais.

Palavras chaves - infográfico; aprendizado suportado por computador; estilos de aprendizagem; satisfação.

\section{Infographics versus Traditional Learning Materials: An Empirical Investigation}

\begin{abstract}
Infographic is a popular type of information visualization that makes use of visual appeal for sharing information faster and effectively. However, there are few empirical studies that investigate how it affects learning process and individuals characteristics such as leaning styles and enjoyment. In this context, this work aims at investigating whether infographics are more effective than traditional learning materials (graphic and text). Thus, we executed an experiment with 54 undergraduate students. The students were distributed between the three types of materials and tested regarding their learning, knowledge retention, and enjoyment. Results showed that infographics were as good as traditional materials for learning and knowledge retention.
\end{abstract}

Keywords - infographic; computer supported learning; learning styles; enjoyment.

\section{Introdução}

A área de Visualização de Informação segue o propósito de apresentar informações que estão em linguagem técnica em um formato de fácil compreensão. Pesquisas têm provado que a associação de imagens a textos influencia positivamente no aprendizado (Mayer, 2014; McLoud, 2000). Os infográficos, por exemplo, são comumente empregados para transformar conceitos abstratos e complexos em conhecimento intuitivo e instantâneo (Smiciklas, 2012). Risch (2008) define-os, de maneira geral, como representações visuais de dados, informações ou conhecimento que podem empregar símbolos para ilustrar ideias. Para serem efetivos, os infográficos devem ser desenvolvidos pensando-se na leitura rápida e na fácil compreensão. Um bom infográfico tem um público-alvo bem definido e pode ser ligado às diferentes áreas da ciência e negócios, desde conceitos de química e física, a conhecimentos gerais como política, meio ambiente e alimentação. É essencial que um infográfico possua representações de dados, como gráficos, e ilustrações adaptadas às explanações textuais 
curtas e objetivas. A Figura 1 mostra um exemplo de infográfico que apresenta como tema o consumo de água na produção de bens de consumo. Nela é possível notar o design remetendo ao tema água (a), o uso de elementos gráficos representando os dados (b), o uso de ilustrações (c) e de curtas descrições textuais complementando as informações (d).

Os estilos de aprendizagem e perfil cognitivo do indivíduo podem afetar a interpretação dos infográficos, prejudicando a construção do conhecimento se essa abordagem de ensino não for adequada ao estilo de aprendizagem (Haider, 2010; Sangineto, 2008). Felder e Silverman (1988) consideram que o processo de aprendizagem envolve a recepção e o processamento de informação e concebem que o estilo de aprendizagem diz respeito à forma como o indivíduo prefere receber e processar essa informação. De Belo (1990) acrescenta a etapa de memorização, esclarecendo que estilo de aprendizagem é "a maneira pela qual a pessoa absorve, processa e retêm a informação".

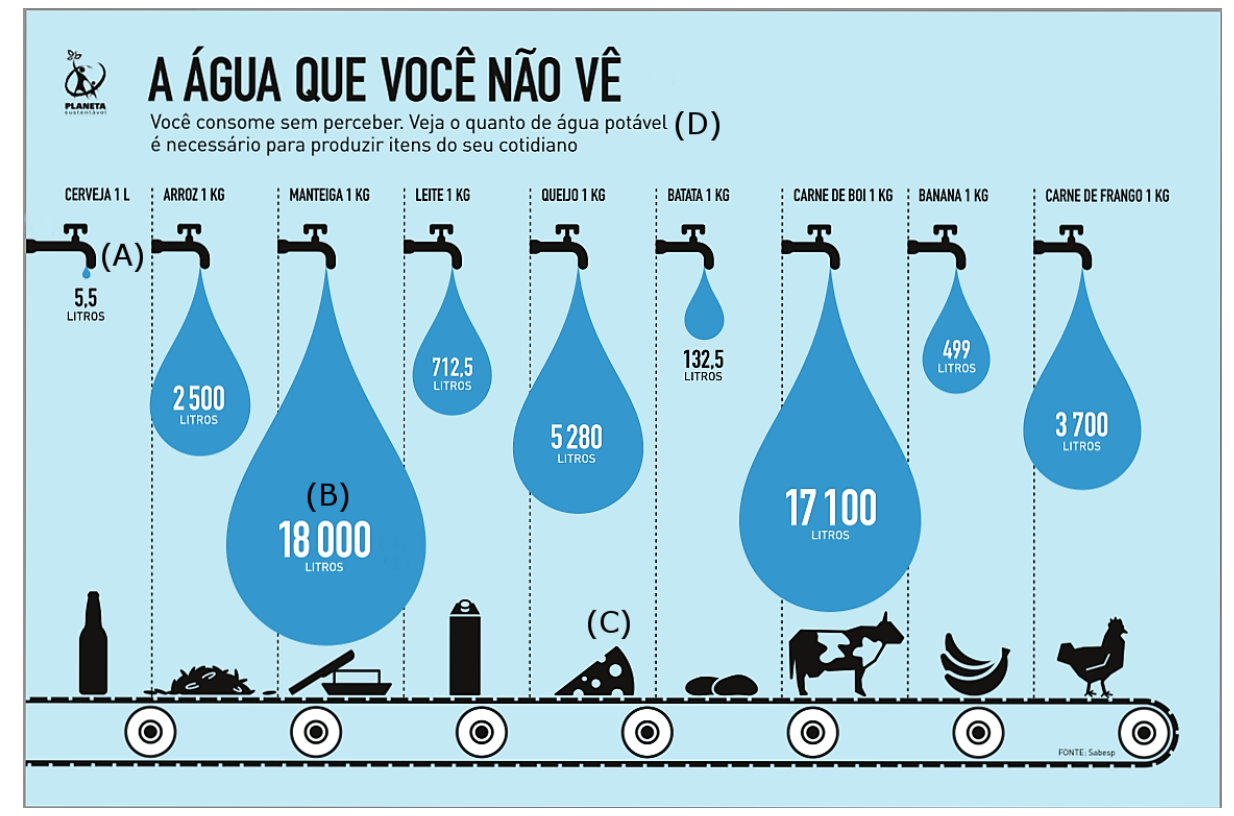

Figura 1: Exemplo de infográfico. Fonte: http://planetasustentavel.abril.com.br/

No contexto educacional, pesquisadores também têm reforçado a importância da motivação, emoção e outros elementos afetivos no processo de aprendizagem (Vail, 1994; John, 2006). É conhecido que induzir uma emoção negativa em um indivíduo prejudica seu desempenho em tarefas cognitivas, enquanto que emoções positivas beneficiam a execução dessas tarefas (Goleman, 1996; Izard, 1984).

Apesar do uso popular dos infográficos e do recente uso como material de aprendizagem (Toth, 2013; Wilkes e Hodson, 2013; Çifçi, 2016), observa-se a falta de estudos que apresentem resultados empíricos sobre a interferência desse tipo de visualização no processo de aprendizagem. Assim, este trabalho descreve a execução e discute os resultados de um experimento controlado, no qual o objetivo é comparar o aprendizado e a retenção de conhecimento de alunos que utilizaram infográficos com o aprendizado e retenção de alunos que utilizaram os formatos tradicionais: gráficos e texto. O experimento foi executado com 54 alunos de graduação e aconteceu em três fases seguindo a metodologia de Wohlin et al. (2012). Além disso, uma vez que infográficos associam imagens e textos para atingir o apelo visual desejado, foi também investigada a interferência dos fatores estilos de aprendizagem e satisfação dos alunos ao interagir com o material. 
Este artigo está organizado da seguinte forma: A Seção 2 apresenta os trabalhos relacionados que utilizam infográficos como material de ensino. A Seção 3 descreve a execução do experimento. Os resultados e as discussões são apresentados na Seção 4. Por fim, são apresentadas as conclusões na Seção 5.

\section{Trabalhos Relacionados}

Devido a sua natureza informativa, os infográficos passaram a ser empregados como material de apoio ao ensino. Nesse contexto, os trabalhos de Toth (2013) e Wilkes e Hodson (2013) reportam as experiências dos autores ao utilizar o desenvolvimento de infográficos como exercício para apoiar o ensino. Os estudantes foram orientados a resumir o conteúdo das aulas na forma de infográficos para assimilar melhor as informações. Apesar de, em ambos os trabalhos, os autores elogiarem essa abordagem e os alunos relatarem maior engajamento no aprendizado, observou-se que não foi aplicado nenhum teste para mensurar o ganho de aprendizagem. Além disso, não foram feitas comparações com outros tipos de materiais. Do mesmo modo, Sudakov et al. (2014) aplicaram um questionário com alunos de graduação e identificaram que $80 \%$ deles foram favoráveis à introdução desse tipo de visualização no processo educacional. Os alunos julgaram que infográficos são mais relevantes para os cursos de Química, Física, Biologia e Ciência da Computação, porém, menos úteis para os cursos de ciências sociais como Sociologia, Filosofia e Psicologia.

Considerando o uso de métricas e processos de avaliação quantitativos, Diakopoulos et al. (2011) exploraram o uso de infográficos com diferentes níveis de elementos de jogos no ensino. Os autores avaliaram o aprendizado, o número de insights e a satisfação do aluno com o infográfico. No entanto, essas variáveis não foram avaliadas em função do material de aprendizagem, mas considerando os níveis de elementos de jogos nos infográficos. Os autores concluíram que quanto menos elementos de jogos, maior o aprendizado, o número de insigths e a satisfação dos alunos, mas não puderam concluir se existe diferença entre empregar infográficos e outros tipos de materiais.

Çifçi (2016) propôs e executou um experimento com o objetivo de comparar o aprendizado de uma turma que utilizou infográficos com uma turma que utilizou materiais tradicionais. Ao final do curso, os alunos que utilizaram infográficos acertaram mais questões em um teste de conhecimento do que os alunos que utilizaram materiais tradicionais. Esse trabalho deixou muitas questões em aberto, por exemplo: a influência do professor, as possíveis diferenças no conteúdo ministrado para os dois grupos, a porcentagem do conteúdo que foi apresentado na forma de infográfico, entre outros.

Realizando um experimento controlado, Zhang-Kennedy et al. (2013) evidenciaram o poder de persuasão dos infográficos. Os autores prepararam três infográficos para informar aos sujeitos como os hackers realizam ataques de segurança (password guessing attacks) e a importância de se criar uma senha forte para seus serviços online. $\mathrm{O}$ estudo concluiu que os sujeitos retiveram significativamente mais informação quando usaram infográficos comparado com o material em forma de texto puro. Ademais, os infográficos foram considerados mais úteis, fáceis de memorizar e persuasivos.

Os trabalhos apresentados nesta seção caracterizam a área de pesquisa sobre "infográficos na educação". Assim, destacam-se que ainda existem poucos trabalhos que investiguem o real impacto desse tipo de visualização no aprendizado e na retenção de conhecimento. Além disso, nenhum dos trabalhos mencionados verifica a interferência de fatores ligados aos sujeitos como o estilo de aprendizagem e satisfação. Observando que infográficos podem ser considerados a combinação de imagens e 
textos, é possível investigar se as conclusões feitas por Mayer (2014) sobre a efetividade da combinação entre informação visual e verbal também podem ser aplicadas aos infográficos.

\section{Experimento}

Esta seção apresenta o planejamento e a condução do experimento, seguindo a metodologia para experimentação proposta por Wohlin et al. (2012). Essa metodologia é composta pela definição do experimento, planejamento, execução e análise. Seguindo a filosofia de dados abertos discutidos em Isotani e Bittencourt (2015), todo material utilizado, incluindo o protocolo do experimento, o material de aprendizagem, os dados coletados, as análises estatísticas, o código fonte do sistema desenvolvido, dentre outros, está publicamente disponível em: http://kamilalyra.github.io/ExperimentoInfograficos/.

\subsection{Definição e Hipóteses}

O objetivo do experimento foi analisar infográficos com o propósito de verificar sua efetividade quando utilizados como material de ensino, considerando seu impacto no aprendizado e na retenção do conhecimento, do ponto de vista do pesquisador no contexto de alunos de graduação em uma sessão de aprendizado apoiada por computador. Essa definição foi baseada em duas questões de pesquisa principais:

QP1. O aprendizado adquirido por meio da utilização de infográficos é maior do que o aprendizado adquirido pela utilização de gráficos e/ou texto?

QP2. O conhecimento retido por meio da utilização de infográficos é maior do que o conhecimento retido pela utilização de gráficos e/ou texto?

A partir dessas questões de pesquisa, dois conjuntos de hipóteses foram formulados e são apresentadas a seguir. As hipóteses nulas $\left(\mathrm{H}_{0}\right)$ são aquelas que se deseja rejeitar para que as hipóteses alternativas $\left(\mathrm{H}_{1}\right)$ sejam automaticamente aceitas.

Hipóteses QP1: $H_{0}: \mu\left(A_{i}\right)=\mu\left(A_{g}\right)=\mu\left(A_{t}\right) \quad H_{1}: \mu\left(A_{i}\right)>\mu\left(A_{g}\right)>\mu\left(A_{t}\right)$

Hipóteses QP2: $H_{0}: \mu\left(C_{i}\right)=\mu\left(C_{g}\right)=\mu\left(C_{t}\right) \quad H_{1}: \mu\left(C_{i}\right)>\mu\left(C_{g}\right)>\mu\left(C_{t}\right)$

Para os dois conjuntos, $A_{n}$ representa o aprendizado e $C_{n}$ representa o conhecimento retido, para $n \in\{$ infográfico, gráfico, texto $\}$ e, $\mu(x)$ refere-se a média dos valores de $x$, para $x \in\left\{A_{n}, C_{n}\right\}$.

\subsection{Materiais e Medidas}

Nesta seção, são descritos os instrumentos utilizados para a condução do experimento.

Materiais de Aprendizagem e Testes de conhecimento: Para chegar aos três tipos de materiais de aprendizagem comparados neste trabalho, foram selecionados 15 infográficos com temas variados. Os infográficos abrangeram temas de conhecimento geral que vão de nutrição a meio ambiente. Essa seleção de domínio foi feita na tentativa de dissipar conhecimentos prévios que possam favorecer determinados sujeitos.

Cada infográfico foi convertido para dois formatos tradicionais: 1) Texto: apresenta todas as informações presentes no infográfico em formato textual, não utilizando nenhum recurso ilustrativo. A Figura 2 apresenta a conversão para texto correspondente ao infográfico apresentado na Seção 1. 2) Gráfico: associa textos a gráficos simples. Todas as ilustrações e elementos de design foram removidos, e toda informação visual foi transcrita, mantendo as representações gráficas para dados. A Figura 3 apresenta a conversão para gráfico+texto correspondente ao infográfico apresentado na Seção 1. O processo de conversão para os dois formatos foi revisado e validado por especialistas para garantir que exatamente as mesmas informações 
presentes nos infográficos poderiam ser retiradas dos gráficos e textos. A partir do conteúdo de cada infográfico foram, escritas três questões, totalizando 45 questões para o questionário de conhecimento aplicado nas três fases do experimento.

\begin{tabular}{|l|}
\hline \multicolumn{1}{|c|}{ A água que você não vê } \\
Você consome sem perceber. Veja o \\
quanto de água potável é necessário para \\
produzir itens do seu cotidiano: \\
- Cerveja $(1 \mathrm{litro})-5,5$ Litros; \\
- Arroz $(1 \mathrm{~kg})-2.500$ Litros; \\
- Manteiga $(1 \mathrm{~kg})-18.000$ Litros; \\
- Leite $(1 \mathrm{~kg})-712,5$ Litros; \\
- Queijo $(1 \mathrm{~kg})-5.280$ Litros; \\
- Batata $(1 \mathrm{~kg})-132,5$ Litros; \\
- Carne de boi $(1 \mathrm{~kg})-17.100$ Litros; \\
- Banana $(1 \mathrm{~kg})-499$ Litros; \\
- Carne de frango $(1 \mathrm{~kg})-3.700$ Litros. \\
\hline
\end{tabular}

Figura 2: Formato textual do infográfico "A água que você não vê".

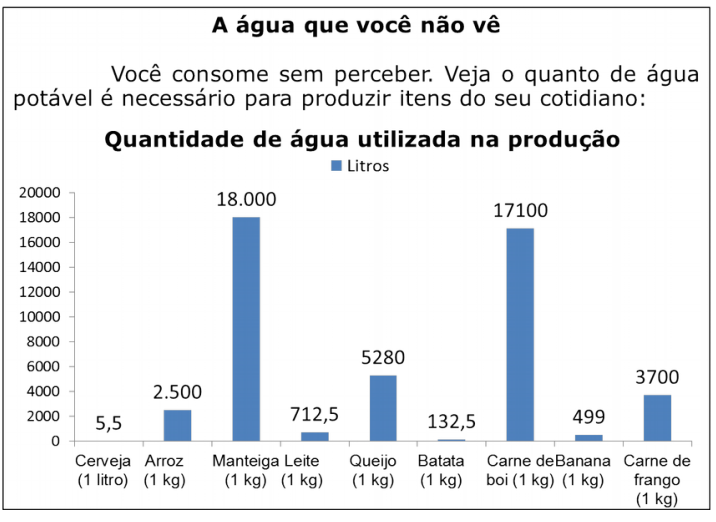

Figura 3: Formato gráfico+texto do infográfico "A água que você não vê".

Os 15 infográficos selecionados, suas respectivas conversões para gráfico e texto, e o questionário de conhecimento podem ser encontrados em http://kamilalyra.github.io/Experimento-Infograficos/.

Self-Assessment Manikin (SAM): A escala SAM (Lang, 1980) para a dimensão de prazer foi utilizada durante a Fase 2 (Pós-teste) para medir a satisfação dos sujeitos durante a interação com o material de aprendizagem. Essa escala é ilustrada por cinco desenhos (manequins) que simbolizam as diferentes gradações de prazer. Desse modo, os sujeitos podem escolher entre um ponto que representa um manequim na escala (valores ímpares), ou um ponto entre dois manequins (valores pares), resultando em nove possibilidades de resposta, como mostrado na Figura 4.

New Index of Learning Style (New-ILS): Neste trabalho, tem-se interesse em identificar o aluno segundo o canal pelo qual ele prefere receber informações: visual ou verbal, considerando que pode haver interação entre essa preferência e o material de aprendizagem que lhe fora designado para a Fase 2 (Pós-teste). Para identificar esse canal, foi utilizado o questionário traduzido e validado por Vieira Junior (2012), o NewILS. Esse questionário é uma versão reduzida do tradicional Index of Learning Style (ILS) (Felder e Silverman, 1988).

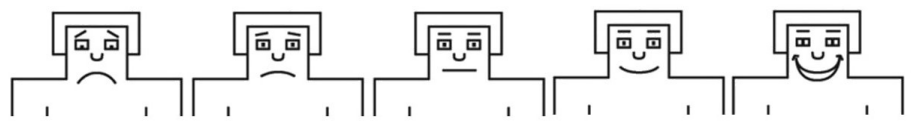

Figura 4: Dimensão prazer da escala SAM. Fonte: Lang, 1980.

Plataforma Web: A plataforma utilizada pelos sujeitos para a realização do experimento foi desenvolvida com base no código aberto do Ambiente Virtual de Aprendizagem E-Game (Pedro e Isotani, 2016). As funcionalidades foram adaptadas e implementadas para cumprir com os objetivos do experimento. Assim, a plataforma foi dividida em três módulos (um para cada fase do experimento). Sua documentação e código fonte podem ser encontrados em http://kamilalyra.github.io/ExperimentoInfograficos/.

\subsection{Seleção dos Sujeitos}

Os participantes do experimento foram selecionados pela conveniência (proximidade entre os autores e os sujeitos no contexto da universidade), caracterizando uma amostra não probabilística (Wohlin et al. 2012). No total, foram selecionados 54 
alunos com idade média de 18,2 $(\sigma=1,22)$ anos de uma turma do primeiro ano do curso de Engenharia da Computação da Universidade de São Paulo. O grupo de sujeitos não possuía formação superior prévia e era constituído de 50 homens e 4 mulheres.

\subsection{Método}

O experimento foi composto de três fases, distribuídas ao longo de três semanas, sendo exatamente sete dias de intervalo entre as fases. A seguir, são descritas as atividades executadas pelos sujeitos em cada uma das fases. Todas as atividades foram cumpridas no computador, com o apoio da plataforma web desenvolvida.

Fase 1 (Pré-teste): Nessa fase, todos os alunos utilizaram o módulo "Pré-teste" da plataforma web. Primeiramente, os sujeitos realizaram um cadastro no sistema informando dados demográficos como idade, sexo e escolaridade. Após realizarem o login no sistema, os sujeitos responderam ao questionário de conhecimento (45 questões) com o objetivo de mensurar seus conhecimentos prévios sobre os temas abordados pelos materiais de aprendizagem. Dessa maneira, foi possível comparar a diferença de conhecimento dos sujeitos entre as Fases 1 e 2, ou seja, antes e depois da interação com as visualizações. Além disso, foi aplicado um segundo questionário para identificar os estilos de aprendizagem (visual ou verbal) dos alunos, o New-ILS (Vieira Junior, 2012). Essa fase teve duração de 50 minutos. Os alunos que finalizaram antes desse tempo foram instruídos a esperar até que todos terminassem.

Fase 2 (Pós-teste): O módulo utilizado nessa fase foi dividido em três versões contendo as mesmas atividades e diferem apenas no tipo de material de aprendizagem carregado. Cada sujeito foi distribuído aleatoriamente entre um dos tipos de material de aprendizagem. No total 18 alunos utilizaram infográficos, 19 utilizaram gráficos e 17 trabalharam com textos. O fluxo das atividades executadas nessa fase está ilustrado na Figura 5. Cada sujeito iniciou essa fase realizando sua autoavaliação de satisfação, utilizando a escala $S A M$. Logo após, foi iniciada a interação com os materiais de aprendizagem, quando o sujeito visualizou um material, e em seguida, respondeu a uma questão sobre o tema do material exibido. A cada três visualizações e questões-tema, o aluno era questionado novamente sobre sua satisfação com a atividade. Esse fluxo foi repetido por cinco vezes, até que o sujeito visualizasse os 15 materiais e respondesse às 15 questões-tema.

Imediatamente após essa interação com os materiais, os sujeitos responderam ao questionário de conhecimento (45 questões) a fim de mensurar o conhecimento adquirido. Por fim, os sujeitos respondem mais uma vez sua autoavaliação de satisfação. Essa fase foi executada em no máximo 120 minutos. Os alunos que finalizaram antes desse tempo foram instruídos a esperar até que todos terminassem.

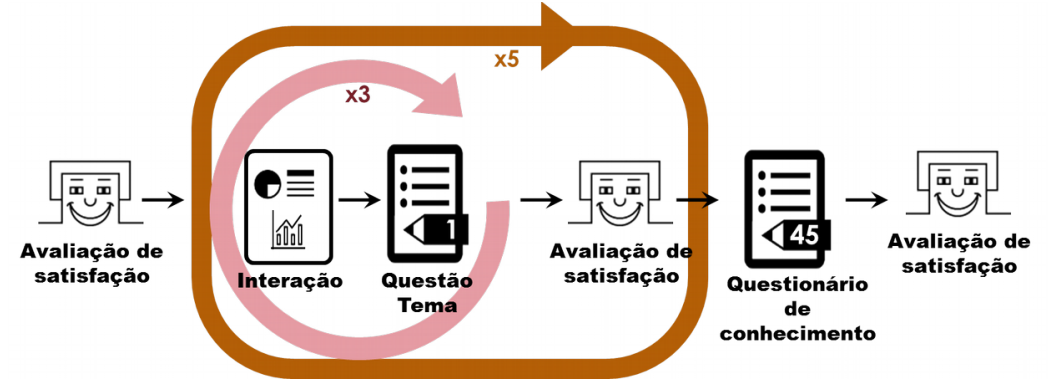

Figura 5: Fluxo de atividades da Fase 2 do experimento (Pós-teste).

Fase 3 (Teste de Retenção): Na última fase do experimento, todos os alunos utilizaram o módulo "Retenção" da plataforma, no qual responderam ao questionário de conhecimento (45 questões) para avaliar o quanto eles retiveram de conhecimento sete dias após a interação que ocorreu durante a Fase 2. Essa fase foi executada em no 
máximo 30 minutos. Os alunos que finalizaram antes desse tempo foram instruídos a esperar até que todos terminassem.

\section{Resultados}

Após a execução das três fases do experimento, foi dado início à verificação e análise dos dados. Para realizar as análises, foi utilizado o software estatístico $\mathrm{R}^{1}$.

\subsection{Estatística Descritiva}

A Tabela 2 apresenta as médias por grupo para todas as variáveis quantitativas, sem considerar os estilos de aprendizagem (Agregado) e separando os sujeitos entre visuais e verbais. A Figura 6 ilustra o número médio de acertos em cada fase por grupo. O grupo que utilizou infográficos apresentou a menor média no pós-teste $\left(\mu_{i}=35,94, \mu_{g}=37,41, \mu_{t}=37,21\right)$ e no teste de retenção $\left(\mu_{i}=33,33, \mu_{g}=34,59, \mu_{t}=34,11\right)$ e, consequentemente, a menor média de aprendizado entre os grupos $\left(\mu_{i}=15,61, \mu_{g}=18,35, \mu_{t}=18,26\right)$. No entanto, os alunos que utilizaram infográficos apresentaram a maior média para retenção de conhecimento $\left(\mu_{i}=-2,61, \mu_{g}=-2,82, \mu_{t}=-3,11\right)$, como apresentado na Figura 8 . Observando a variável tempo médio gasto em cada material, o grupo que utilizou infográficos foi o que passou menos tempo interagindo com o material, enquanto que o grupo de gráficos foi o que gastou mais tempo $\left(\mu_{i}=91,3, \mu_{g}=120,55, \mu_{t}=114,86\right)$, como ilustrado na Figura 7.

Tabela 1: Estatística descritiva das variáveis controladas.

\begin{tabular}{|c|c|c|c|c|c|c|c|}
\hline & \multirow[b]{3}{*}{ Tratamento } & \\
\hline & & \multicolumn{2}{|c|}{ Agregado } & \multicolumn{2}{|l|}{ Visual } & \multicolumn{2}{|l|}{ Verbal } \\
\hline & & Média & Desvio padrão & Média & Desvio padrão & Média & Desvio padrão \\
\hline \multirow{3}{*}{$\begin{array}{l}\text { Número de } \\
\text { acertos no Pré- } \\
\text { teste (A) }\end{array}$} & Infográfico & 20,33 & 3,48 & 20,07 & 3,73 & 21,67 & 1,53 \\
\hline & Gráfico & 19,06 & 3,23 & 19,09 & 3,83 & 19 & 2 \\
\hline & Texto & 18,95 & 2,38 & 19,4 & 2,8 & 17,25 & 1,26 \\
\hline \multirow{3}{*}{$\begin{array}{l}\text { Número de } \\
\text { acertos no Pós- } \\
\text { teste (B) }\end{array}$} & Infográfico & 35,94 & 3,73 & 36,2 & 3,59 & 34,67 & 5,03 \\
\hline & Gráfico & 37,41 & 4,66 & 37,64 & 4,84 & 37 & 4,73 \\
\hline & Texto & 37,21 & 6,54 & 37 & 7,39 & 38 & 0,82 \\
\hline \multirow{3}{*}{$\begin{array}{l}\text { Número de } \\
\text { acertos na } \\
\text { Retenção (C) }\end{array}$} & Infográfico & 33,33 & 3,61 & 33,53 & 4,39 & 32,33 & 6,66 \\
\hline & Gráfico & 34,59 & 5,88 & 35,18 & 5,64 & 33,5 & 6,72 \\
\hline & Texto & 34,11 & 5,15 & 33,8 & 5,78 & 35,25 & 0,96 \\
\hline \multirow{3}{*}{$\begin{array}{l}\text { Aprendizado } \\
(\mathbf{B}-\mathbf{A})\end{array}$} & Infográfico & 15,61 & 3,47 & 16,13 & 3,14 & 13 & 4,58 \\
\hline & Gráfico & 18,35 & 5,52 & 18,55 & 5,82 & 18 & 5,44 \\
\hline & Texto & 18,26 & 6,93 & 17,6 & 7,66 & 20,75 & 2,06 \\
\hline \multirow{3}{*}{$\begin{array}{l}\text { Retenção (C - } \\
\text { B) }\end{array}$} & Infográfico & $-2,61$ & 1,88 & $-2,66$ & 1,84 & $-2,33$ & 2,52 \\
\hline & Gráfico & $-2,82$ & 2,92 & $-2,45$ & 3,08 & $-3,5$ & 2,74 \\
\hline & Texto & $-3,11$ & 2,73 & $-3,2$ & 3,08 & $-2,75$ & 0,5 \\
\hline \multirow{3}{*}{$\begin{array}{l}\text { Satisfação } \\
\text { (min: 1; max: } \\
\text { 9) }\end{array}$} & Infográfico & 5,24 & 1,84 & 5,44 & 1,59 & 4,24 & 3,03 \\
\hline & Gráfico & 5,84 & 1,53 & 6,09 & 1,66 & 5,38 & 1,25 \\
\hline & Texto & 5,01 & 1,99 & 4,73 & 2,07 & 6,04 & 1,43 \\
\hline \multirow{3}{*}{$\begin{array}{l}\text { Tempo de } \\
\text { visualização } \\
\text { (segundos) }\end{array}$} & Infográfico & 91,3 & 40,17 & 96,65 & 37,98 & 64,41 & 48,14 \\
\hline & Gráfico & 120,55 & 59,26 & 125,85 & 66,21 & 110,83 & 47,88 \\
\hline & Texto & 114,86 & 54,07 & 112,77 & 55,65 & 122,68 & 54,68 \\
\hline
\end{tabular}




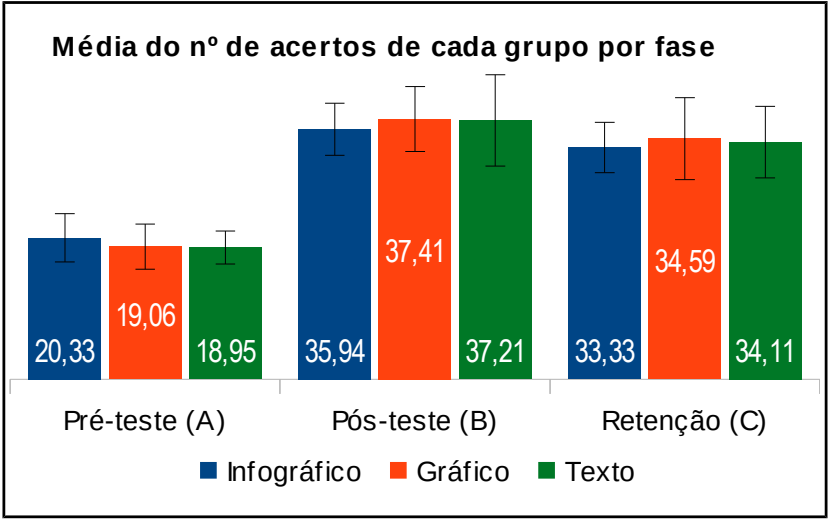

Figura 6: Média no número de acertos em cada fase por grupo.

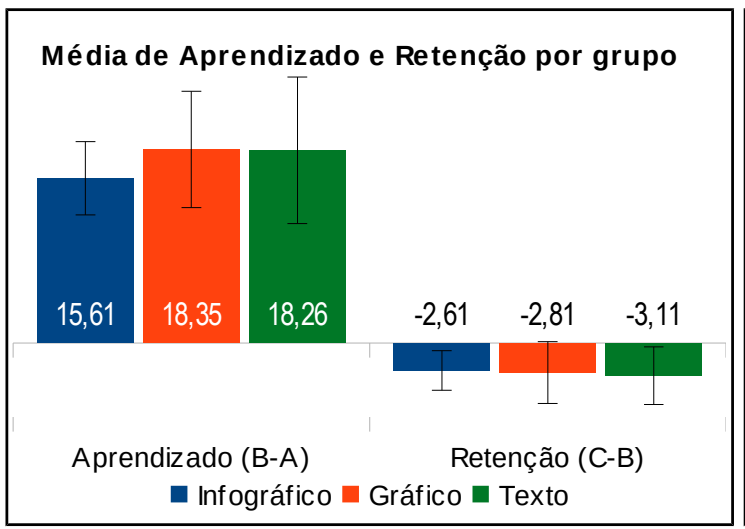

Figura 8: Média de Aprendizado e Retenção por grupo.
Média de tempo de visualização(segundos)

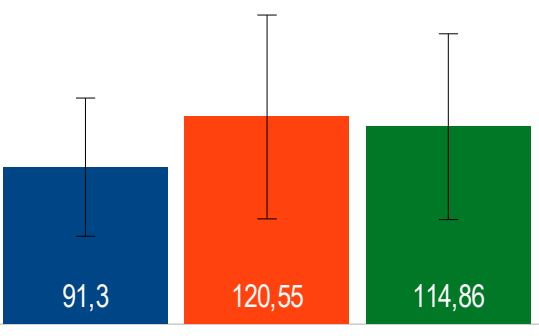

Tempo (s)

- Infográfico Gráfico $\quad$ Texto

Figura 7: Média de tempo de interação com o material por grupo.

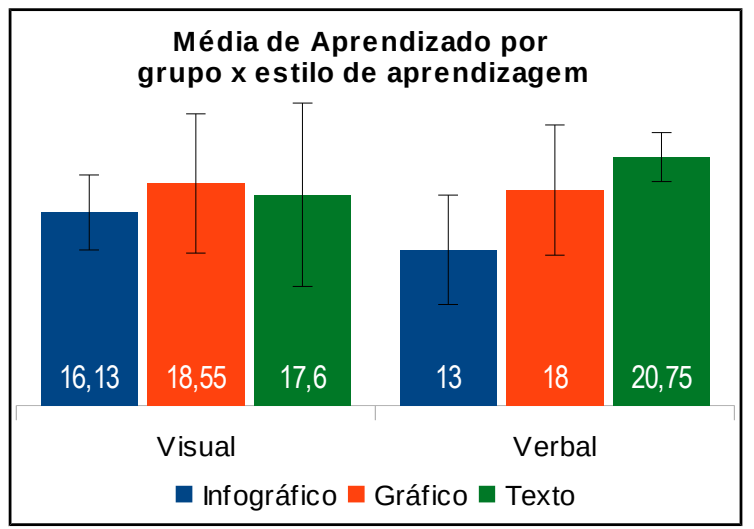

Figura 9: Média de Aprendizado por grupo e estilos de aprendizagem.

\subsection{Correlações entre variáveis}

Além da comparação das médias entre os grupos, foram calculadas as correlações entre as variáveis medidas utilizando o coeficiente de correlação de Pearson ${ }^{2}$. Para o grupo de alunos que usou infográficos, foram identificadas correlações significativas entre as seguintes variáveis: tempo $\mathrm{x}$ acertos no pós-teste $(r=0,688, p<0,001)$, tempo $\mathrm{x}$ acertos no teste de retenção $(r=0,556, p<0,05)$ e tempo $\mathrm{x}$ aprendizado $(r=0,553, p<0,05)$.

Do mesmo modo, o grupo de alunos que utilizou texto também apresentou correlação entre: tempo x acertos no pós-teste $(r=0,656, p<0,001)$, tempo $\mathrm{x}$ acertos no teste de retenção $(r=0,702, p<0,001)$ e tempo $\mathrm{x}$ aprendizado $(r=0,691, p<0,001)$. Além disso, esse grupo foi o único que apresentou correlação entre a variável satisfação e acertos no pós-teste $(r=0,503, p<0,05)$ e no teste de retenção $(r=0,651, p<0,001)$. Considerando os alunos que utilizaram gráficos, foi encontrada correlação apenas entre as variáveis tempo $\mathrm{x}$ retenção de conhecimento $(r=0,602, p<0,05)$.

\subsection{Testes de Hipótese}

Para testar as hipóteses formuladas na Seção 3.1, foi utilizado o teste ANOVA (Analysis of Variance), que é recomendado para configurações experimentais nas quais existem um fator (material de aprendizagem) e mais de dois tratamentos (infográfico,

2 Mede o grau de correlação entre duas variáveis e assume valores entre -1 e +1: 1 significa correlação perfeita positiva entre as duas variáveis, -1 significa correlação perfeita negativa (o acréscimo de uma implica em decréscimo de outra) e 0 significa não dependência linear entre as variáveis. 
gráfico e texto), e nas quais as métricas das variáveis dependentes (aprendizado e conhecimento retido) apresentam medidas na escala razão. No contexto desse experimento, o teste ANOVA mostrou se existe diferença significativa ${ }^{3}$ entre as médias de aprendizado e de retenção, em função dos materiais de aprendizagem utilizados. $\mathrm{O}$ resultado do teste $A N O V A$ para os dois conjuntos de hipóteses foi:

Aprendizado: Dado o valor não significativo no teste ANOVA realizado entre a média de aprendizado dos grupos $(p=0,249)$, não é possível negar a hipótese nula. Consequentemente, não se pode aceitar a hipótese de que o aprendizado por meio de infográficos é maior do que pelos materiais tradicionais (gráfico e texto).

Retenção: Do mesmo modo como ocorreu para aprendizagem, não existe diferença significativa entre as médias de retenção dos grupos, isso é comprovado pelo valor resultante do teste ANOVA $(p=0,839)$. Assim, também não é possível afirmar que os infográficos beneficiam a retenção do conhecimento quando comparado com gráfico e texto.

\subsection{Discussão}

Pode-se considerar a média de aprendizado e de retenção entre os grupos, ilustrada pela Figura 8, um indício de que o uso de infográficos como material de aprendizagem favorece mais a retenção de conhecimento do que o aprendizado imediato. Isso pode estar associado ao fato de que à primeira vista, os infográficos podem ser menos compreensíveis do que formatos tradicionais que têm uma sequência linear na apresentação dos fatos. No entanto, os infográficos possuem um design mais apelativo e menos linear, favorecendo a memória quando comparado aos textos (Lyra et al., 2016).

Nota-se que alunos visualizando infográficos apresentaram a menor média de tempo de interação com os materiais, como ilustrado pela Figura 7. É possível supor que o tempo menor é decorrente da facilidade de se ler e interpretar os infográficos, fazendo com que os alunos assimilem rapidamente todas as informações. Outra possibilidade é de que os infográficos são menos lineares e assim, mais complexos de serem lidos, deixando os alunos desmotivados em explorar a visualização, passando mais rapidamente para o próximo infográfico. Na tentativa de verificar qual das suposições é verdadeira, observamos o valor médio de satisfação. Se os alunos passaram mais rápido pelos infográficos, mas deram menores valores na escala $S A M$, presume-se que eles ficaram desmotivados. Em contrapartida, se os valores da escala $S A M$ forem maiores, é mais provável que os alunos tenham encontrado mais facilmente as informações nos infográficos. No entanto, as médias de satisfação entre os grupos foram tão semelhantes que não foi possível extrair as informações desejadas $\left(\mu_{i}=5,24, \mu_{g}=5,84, \mu_{t}=5,01\right)$.

Considerando os estilos de aprendizagem, pode-se destacar diferenças no comportamento das médias entre os alunos visuais e verbais. De acordo com a definição de Felder e Silverman (1988), alunos Visuais gravam mais informações por meio de imagens, gráficos, quadros e filmes, enquanto que aprendizes do tipo Verbais preferem ouvir ou escrever as informações. Logo, é esperado que a aprendizagem dos alunos visuais seja favorecida ao utilizarem infográficos. Pode-se confirmar esse comportamento ao comparar as médias de acertos (pós-teste e teste de retenção) e de aprendizado. Por meio da Figura 9, nota-se que dentre os alunos utilizando infográficos, os visuais tiveram médias maiores $\operatorname{Vis}\left(\mu_{i}=16,13\right)$ do que os alunos verbais $\operatorname{Ver}\left(\mu_{i}=13\right)$. O contrário ocorreu para os alunos utilizando texto $\operatorname{Vis}\left(\mu_{t}=17,6\right)$ $\operatorname{Ver}\left(\mu_{t}=20,75\right)$.

Não foi apenas a aprendizagem que foi favorecida pela associação de estilos de 
aprendizagem e material de ensino. A média de satisfação dos alunos visuais que usaram infográficos $\operatorname{Vis}\left(\mu_{i}=5,44\right)$ foi maior comparada à dos alunos verbais com o mesmo tipo de material $\operatorname{Ver}\left(\mu_{i}=4,24\right)$. Para o grupo de alunos que usou texto puro, a média de satisfação foi maior para os alunos verbais $\operatorname{Ver}\left(\mu_{i}=6,04\right)$ do que para os visuais $\operatorname{Vis}\left(\mu_{i}=4,73\right)$. Essas diferenças ocorrem de modo coerente com a teoria de Felder e Silverman (1988) que concebem estilo de aprendizagem como a forma como o indivíduo prefere receber e processar informações.

Ao analisar o grupo de alunos que utilizou infográficos pode-se identificar uma correlação positiva forte entre o tempo gasto em cada infográfico e o número de acertos no pós-teste e o número de acertos no teste de retenção. Essa correlação indica que quanto mais tempo os alunos gastaram visualizando os infográficos, maior foi sua aprendizagem. Igualmente ocorreu para o grupo de alunos que utilizou texto. Além disso, para esse grupo é possível relacionar positivamente o número de acertos no pósteste e no teste de retenção com a média de satisfação declarada pelo aluno. Assim, quanto mais positivo o valor na escala $S A M$, maior o número de acertos.

Por fim, para os três grupos investigados (infográfico, gráficos e texto), identificou-se uma forte correlação entre o tempo médio de visualização e a retenção de conhecimento, ou seja, quanto mais tempo os alunos gastaram estudando os materiais, mais informações eles foram capazes de lembrar uma semana após a interação com os materiais. Resultado consistente com a literatura na área (Mayer, 2014).

\section{Conclusões}

Existem poucos trabalhos na literatura que investiguem empiricamente o uso de infográficos como material de aprendizagem. Nesse contexto, este artigo descreveu a execução de um experimento controlado realizado com 54 alunos de graduação. $\mathrm{O}$ objetivo do experimento foi comparar o aprendizado e a retenção dos alunos que utilizaram infográficos com as formas tradicionais: gráfico e texto puro. $\mathrm{O}$ experimento também mediu a influência de fatores individuais como estilos de aprendizagem e satisfação com o material. Os resultados não permitiram afirmar que existe diferença significativa de aprendizado e retenção de conhecimento entre os três grupos. Dessa forma, os infográficos demonstraram ser um formato tão bom quanto os formatos de materiais de aprendizagem tradicionais. Contudo, foram encontrados indícios de que os infográficos podem trazer mais benefícios para a retenção do conhecimento do que para a aprendizagem imediata. Assim, sugere-se que o objetivo do ensino seja analisado antes de se optar pelo uso de materiais de aprendizagem na forma de infográficos.

Finalmente, foi possível identificar a influência dos fatores individuais como satisfação e estilos de aprendizagem. A comparação entre as médias de aprendizado dos grupos visuais e verbais foi condizente com a teoria de Felder e Silvermam (1988), no qual os alunos visuais foram favorecidos pelo formato mais ilustrativo de representação, nesse caso os infográficos. Além do aprendizado, os alunos visuais também apresentaram um grau de satisfação maior ao usarem infográficos do que ao usarem textos.

Sugere-se, como trabalhos futuros, uma nova execução do experimento variando o domínio abordado pelos infográficos e o tipo de processo cognitivo avaliado (lembrar, entender, aplicar, analisar, avaliar ou criar) (Mayer, 2002). Desse modo, seria possível obter dados comparáveis aos resultados apresentados aqui e gerar assertivas concretas sobre o melhor emprego para infográficos.

\section{Agradecimentos}

Os autores agradecem a CNPq, CAPES e FAPESP pelo apoio financeiro. 


\section{Referências Bibliográficas}

ÇIFÇI, T. Effects of Infographics on Students Achievement and Attitude towards Geography Lessons. Journal of Education and Learning, v. 5, n. 1, p. 154-166, 2016.

DE BELLO, T. C. Comparison of eleven major learning styles models: Variables, appropriate populations, validity of instrumentation, and the research behind them. Journal of Reading, Writing, and Learning Disabilities, v. 6, n. 3, p. 203-222, 1990.

DIAKOPOULOS, $\mathrm{N}$; KIVRAN-SWAINE, F; NAAMAN, M. Playable data: characterizing the design space of game-y infographics. In: Proceedings of the SIGCHI Conference on Human Factors in Computing Systems. ACM. Vancouver, BC, Canada. 2011. p. 1717-1726.

FELDER, R. M.; SILVERMAN, L. K. Learning and teaching styles in engineering education. Engineering education, v. 78, n. 7, p. 674-681, 1988.

GOLEMAN, D. Emotional Intelligence: Why It Can Matter More than IQ. Learning, v. 24, n. 6, p. 49-50, 1996.

HAIDER, M.; SINHA, A.; CHAUDHARY, B. An Investigation of relationship between learning styles and performance of learners. International Journal of Engineering Science and Technology, v. 2, n. 7, p. 2813-2819, 2010.

ISOTANI, S.; BITTENCOURT, I. I. Dados Abertos Conectados: Em busca da Web do Conhecimento. Editora Novatec. 176p, 2015.

IZARD, C. E. Emotion-cognition relationships and human development. In: IZARD, C. E; KAGAN, J; ZAJONC, R. B. (Ed). Emotions, cognition, and behavior. Cambridge: Cambridge University Press p. 17-37, 1984.

JOHN-STEINER, V. Creative collaboration. New York: Oxford University Press, 2000.

VIEIRA JUNIOR, N. Planejamento de um ambiente virtual de aprendizagem baseado em interfaces dinâmicas e uma aplicação ao estudo de potência elétrica. Tese (doutorado). Universidade Estadual Paulista Júlio de Mesquita Filho, Faculdade de Engenharia de Ilha Solteira. 2012.

LANG, P. J. Behavioral treatment and bio-behavioral assessment: Computer applications. In Technology in mental health care delivery systems, pp. 119-137, 1980.

LYRA, K. T; ISOTANI, S; REIS, R. C. D; MARQUES, L. B; PEDRO, L. Z; JAQUES, P. A; BITTENCOURT, I. I. Infographics or Graphics+Text: Which Material is Best for Robust Learning?. Proceedings of the IEEE 16th International Conference on Advanced Learning Technologies (ICALT), p. 366-370, 2016.

MAYER, R. E. Rote Versus Meaningful Learning. Journal of Theory into practice, v. 41, n. 4, p. 226-232, 2002.

MAYER, R. E. The Cambridge Handbook of Multimedia Learning. Santa Barbara: Cambridge University Press, 930p. 2014.

MCLOUD, S. Reinventing Comics: How Imagination and Technology Are Revolutionizing an Art Form. Perennial, New York, p. 118,122-123, 2000.

PEDRO, L. Z.; ISOTANI, S. Explorando o Impacto da Gamificação na Redução do Gaming the System em um Ambiente Virtual de Aprendizagem. In: Anais do Congresso Brasileiro de Informática na Educação, Uberlandia. p. 81-90, 2016.

RISCH, John S. On the role of metaphor in information visualization. arXiv preprint 
arXiv:0809.0884, 2008.

SANGINETO, E., CAPUANO, N., GAETA, M., E MICARELLI, A. Adaptive course generation through learning styles representation. Universal Access in the Information Society, v. 7, n. 1-2, p. 1-23, 2008.

SMICIKLAS, M. The power of Infographics: Using Pictures to Communicate and Connect With Your Audiences. Indianapolis: Que Publishing, 199p. 2012

SUDAKOV, I., BELLSKY, T., USENYUK, S., E POLYAKOVA, V. Mathematics and Climate Infographics: A Mechanism for Interdisciplinary Collaboration in the Classroom. arXiv preprint arXiv:1405.6435, p. 1-8. 2014.

TOTH, C. Revisiting a Genre Teaching Infographics in Business and Professional Communication Courses. Business Communication Quarterly, v. 76, n. 4, p. 446-457, 2013.

VAIL, P. L. Emotion: The on/off switch for learning. Modern Learning Press, 257p. 1994.

WILKES, G. E HODSON, J. Using social media aggregation and curation techniques in the classroom to identify discourse trends and support brand operations. In IEEE International Professional Communication Conference (IPCC). p. 1-7, 2013.

WOHLIN, C., RUNESON, P., H“OST, M., OHLSSON, M. C., REGNELl, B., E WESSL'EN, A. Experimentation in Software Engineering. Springer Science \& Business Media, 236p. 2012.

ZHANG-KENNEDY, L., CHIASSON, S., E BIDDLE, R. Password advice shouldn't be boring: Visualizing password guessing attacks. In IEEE eCrime Researchers Summit (eCRS), p. 1-11, 2013. 